\title{
Quantifying the Flexibility of Residential Electricity Demand in 2050: a Bottom-Up Approach
}

\author{
Arne van Stiphout, Jonas Engels, Dries Guldentops, Geert Deconinck \\ Research Group ELECTA, Dept. Electrical Engineering (ESAT) \\ KU Leuven \& EnergyVille \\ Heverlee, Belgium \\ arne.vanstiphout@esat.kuleuven.be
}

\begin{abstract}
This work presents a new method to quantify the flexibility of automatic demand response, combined with real time pricing, applied to residential electricity demand using price elasticities. A stochastic bottom-up model of flexible electricity demand in $\mathbf{2 0 5 0}$ is presented. Three types of flexible devices are implemented: electric heating, electric vehicles and wet appliances. Each house schedules its flexible demand w.r.t. a varying price signal, in order to minimize its electricity cost. Own- and cross-price elasticities are obtained through a regression analysis. Via a Monte Carlo approach-based method, the elasticities are scaled up to a country level. The results show that the electric energy demand will double and that, when combining automatic demand response with real time pricing, power peaks in demand could be incurred that are 5 to 8 times greater than today. The elasticity matrices show that for Belgium most flexibility is available in winter and least in summer.
\end{abstract}

Index Terms-Energy consumption, Load modeling, Power system economics, Power system modeling

\section{INTRODUCTION}

In order to limit the global temperature rise to an average of $2{ }^{\circ} \mathrm{C}$, the leaders of the European Union and the G8 announced to reduce greenhouse gas emissions to at least $80 \%$ below 1990 levels by 2050 [1]. The European Commission translated this objective into several roadmaps, including one by the DG Energy [2], implying near carbon neutrality for the power sector in 2050. Several major stakeholders in the energy sector have issued studies with the goal of establishing scenarios for reaching this low-carbon energy system by 2050 [1], [3]. In the outcome of these studies, a significant share of the electricity production in 2050 comes from renewables, ranging between $40 \%$ and $100 \%$. Their variable output and limited predictability result in a need for back-up generation capacity and balancing services [4], [5].

Active participation of the demand side through the use of Demand Response (DR) programs can provide part of this operational flexibility. Several DR programs exist, a good overview is given in [6]. Many economists believe that real time pricing (RTP) is one of the most direct and efficient DR mechanism and should be the focus of policy makers [7].

Different modelling approaches exist to represent this flexibility in energy system models. However, they all suffer from some drawbacks. Some approaches represent the flexibility very coarse as a given percentage of the energy demand that can be shifted [1], [8], [9]. Others lose valuable temporal information [10] or only consider one type of flexible device [11]-[14]. Still others are more economically oriented, representing flexibility by price elasticities. They do not take into account the technical characteristics of different flexible devices [15], [16].

A major challenge for the system operator is to integrate this flexibility ex-ante in the market. To do so, the available flexibility must first be quantified. A new method is presented to quantify the flexibility of automatic demand response combined with real time pricing applied to residential electricity demand using price elasticities. To include the technical characteristics of the flexible devices, the model uses a bottom-up approach. A carbon-neutral scenario is assumed with a high degree of electrification, reflecting a possible state of affairs for 2050. All residential customers are assumed to participate in an RTP program. The model is applied to the Belgian residential electricity demand as extrapolated for 2050 .

The remainder of this paper is organized as follows. First, Section II discusses the models of the flexible devices taken into account and explains the optimization problem. Section III describes the applied regressions in order to come to the elasticity matrices. To find the average values of these elasticity matrices, a Monte Carlo simulation is performed, which is explained in Section IV. The results of this Monte Carlo simulation are presented and discussed in Section V, followed by the conclusion in Section VI.

\section{Demand Model}

The residential electricity demand of a household can be divided in two different types: non-flexible demand and flexible demand, which can be shifted in time. Non-flexible demand and occupancy profiles are modelled based upon [17] and scaled to 2050 according to [9]. Three types of flexible devices are considered in this paper. The models of these devices and the corresponding data and assumptions will be briefly explained in the sections below.

\section{A. Flexible Devices}

Flexible devices can be sudivided into discrete devices and continuous devices. Discrete devices must run a complete predefined cycle once started and cannot be interrupted. Discrete 
devices implemented in this paper are 3 types of wet appliances. Continuous devices are not restricted in their load cycle. They can be interrupted as much as desired and can draw a varying power. They will react to the comfort constraints imposed by the users. Continuous devices implemented in this paper are electric heating and electric vehicles (EVs).

1) Wet Appliances: Wet appliances include washing machines, dishwashers and tumble dryers. Important characteristics of these devices are penetration rates, load cycles, start and stop times and number of cycles per week. All these data for Belgium are taken from the Smart A project [18]. This data contains a set of 5000 load cycles for every type of device. According to the penetration rate, a household may or may not possess a certain type of device.

2) Heat Pump \& Auxiliary Heaters: Each residence is equipped with an air coupled heat pump (HP) that can deliver both space heating ( $\mathrm{SH}$ ) and domestic hot water (DHW). To ensure that the temperature constraints set by the inhabitants can always be met, the HP is backed up with two auxiliary electric heaters AUX1 and AUX2. The building model and the DHW model are both based on [19].

a) Space Heating: The building model consists of one zone, heated by a floor heating system and is represented by an RC model based on [20]. Since this is a linear approach, the thermal behaviour of the building can be described by a linear state space model. The temperature in the houses is influenced by the incoming solar radiation and the ambient temperature. These data are obtained from Meteonorm [21]. The temperature can be adjusted by the use of the heat pump or the auxiliary heaters. Three types of newly built buildings are considered: two types of single family houses and one type of multi family house from [20] and [22].

b) Domestic Hot Water: The DHW is supplied by and stored in a hot water storage tank. The thermal power that needs to be supplied to the hot water tank at each moment depends on the water extracted from the tank. It is assumed that the first two inhabitants use 50 liter per person per day and each next inhabitant consumes an extra 30 liter per day. Per person 2 to 3 tapping moments per day are assumed and the probability of DHW consumption over a day is based on [23]. The water in the tank can only be heated by the heat pump and AUX1.

3) Electric Vehicles: Based on [24] and [25], it is assumed that $71.4 \%$ of the households posses an EV. The EVs' charging moments depend on their driving behaviour. Data of such behaviour is based on [26] and includes 100 different driving patterns for each day. Important parameters include moments when the EV is at home, when it is driving and when it needs a certain amount of energy to drive a certain distance.

\section{B. Optimization Problem}

All devices are grouped into a house according to their penetration rate. A house receives an RTP time series and a central optimizer schedules the flexible devices with the aim of minimizing the electricity cost. Because of the discrete nature of the wet appliances, this results in a mixed-integer linear problem. The constraints of this optimization problem are:
1) Wet Appliances: The load cycle of wet appliances can be shifted between the start and stop times imposed by the user.

2) Space Heating \& Domestic Hot Water: The temperature must always lie between $16^{\circ} \mathrm{C}$ and $22^{\circ} \mathrm{C}$ when occupants are absent and between $20^{\circ} \mathrm{C}$ and $23^{\circ} \mathrm{C}$ when the house is occupied. The thermal power is limited by the maximum equipment ratings for the different heaters.

3) Electric Vehicles: The state of charge of the battery has to be at least $20 \%$. Vehicle-to-grid is not included. The maximum charging power is limited by the household connection.

4) Connection constraint: The total electric power that can be drawn by a dwelling is limited to $9.2 \mathrm{kVA}$ as this is currently the standard for the average household in Flanders.

5) Cyclic constraints: The optimization problem has a finite time horizon. Start and end conditions have to be imposed. Therefore, extra cyclical constraints are imposed which ensure e.g. that all the energy consumed by the EVs and stored in its batteries is also charged within the considered time period.

\section{Flexible Devices}

Several parameters in the model of a house are stochastically defined (see Table I). The number of occupants per household is based on the population structure of Belgium [27]. To represent the penetration rates and the number of occupants per household in a realistic way, multiple houses are created. Following [28], they are grouped together in neighborhoods of 70 houses. The energy consumption of such a neighborhood is optimized in the same way as described in Section II-B.

TABLE I

Stochastic Elements in the Model

\begin{tabular}{r|lr|l}
\multirow{5}{*}{ House } & Number of inhabitants & EV & Penetration \\
Occupancy & & Energy needed \\
Non flexible loads & & Time to charge \\
Wet appliances & Oecupancy & DHW & Hot water demand \\
& Load cycle & SH & Type of house \\
Start \& stop times & PV & Penetration \& size
\end{tabular}

\section{REgRESSION ANALYSIS}

An optimization as performed in Section II leads to a certain electricity demand $q$ given a price $p$ for each time step. These optimizations are repeated for different price signals and lead to multiple $(p, q)$ points. Given these points, a regression can be performed that yields coefficients that express the relationship between price $p$ and demand $q$ at each moment. In this paper, a linear relationship is proposed and the coefficients of the regression are assembled in a so called elasticity matrix $\epsilon_{N \times N}$. This matrix represents the relationship between a change in electricity price $\Delta p$ and a change in demand $\Delta q$, relative to a certain reference point $\left(p_{\text {ref }}, q_{\text {ref }}\right)$ :

$$
\epsilon_{N \times N} \cdot \Delta p=\Delta q
$$

with

$$
\epsilon_{N \times N}=\left(\begin{array}{ccc}
\epsilon_{11} & \cdots & \epsilon_{1 N} \\
\vdots & \ddots & \vdots \\
\epsilon_{N 1} & \cdots & \epsilon_{N N}
\end{array}\right)
$$


and

$$
\begin{aligned}
\Delta p & =\frac{p-p_{\text {ref }}}{p_{\text {ref }}} \\
\Delta q & =\frac{q-q_{\text {ref }}}{q_{\text {ref }}}
\end{aligned}
$$

the relative differences in electricity consumption and price, w.r.t. a reference point on an hourly basis. In this matrix, the own-price and cross-price elasticity are defined as:

$$
\epsilon_{i i} \cdot \Delta p_{i}=\Delta q_{i}, \quad \epsilon_{i j} \cdot \Delta p_{i}=\Delta q_{j}
$$

with $\Delta p_{i}=\left(p_{i}-p_{\text {ref }, i}\right) / p_{\text {ref }, i}$ the relative difference in price w.r.t. a reference price and $\Delta q_{i}=\left(q_{i}-q_{r e f, i}\right) / q_{r e f, i}$ the relative difference in electricity consumption w.r.t. a corresponding reference electricity consumption. These elasticities define a linear relationship between the deviation of a price at a certain hour $j$ from a reference price and the deviation of the associated electricity consumption at the hour $i$.

The time horizon $N$ of prices that influence the electricity use is taken to be 24 as the typical cycle of residential electricity use repeats itself every 24 hours. Later it will be proven that this horizon is sufficient. Special attention has to be paid to the cyclical constraints set in Section II.

\section{A. Data Processing}

The price vectors $p$ used in the optimizations are obtained from Belpex prices of 2012 and 2013 [29]. These prices are rescaled in order to all have the same mean value of 1 . In order to not let them influence the regression, extreme situations or outliers - price vectors with a price more than 3 times the standard deviation of the mean value for that hour - are removed from the sample set. As the optimizations are computationally intensive, a compromise between computational time and the quality of the regression has been made. Only $n=350$ price samples are retained.

\section{B. Least Square Estimate}

To obtain the linear price elasticities, a linear regression is performed that relates a vector $\Delta p$ of size $N$ (24 in the case of one day) to a vector $\Delta q$, also of size $N$. These are in fact $N$ independent regressions for each $\Delta q_{i}, i=1, \ldots, N$ if we assume that each element $\Delta q_{i}$ only depends on $\Delta p$. Hence, $N$ multiple linear regressions are performed using the least squares estimate technique. This means finding a vector $\boldsymbol{\epsilon}_{\mathbf{i}}=\left(\epsilon_{i 1}, \epsilon_{i 2}, \ldots, \epsilon_{i N}\right)$ such that:

$$
\Delta q_{i}^{k}=\boldsymbol{\epsilon}_{\mathbf{i}} \Delta p^{k}+e r r_{i}^{k}
$$

with $\operatorname{err}_{i}^{k}$ the error on the linear model for time step $i$ and sample $k$. If we define:

$$
\begin{aligned}
\mathbf{e r r}_{\mathbf{i}}=\left(\operatorname{err}_{i}^{1}, \ldots, \operatorname{err}_{i}^{n}\right) & i=1, \ldots, N \\
\boldsymbol{\Delta} \mathbf{q}_{\mathbf{i}}=\left(\Delta q_{i}^{1}, \ldots, \Delta q_{i}^{n}\right) & i=1, \ldots, N
\end{aligned}
$$

and

$$
\Delta \mathbf{p}=\left(\begin{array}{ccc}
p_{1}^{1} & \cdots & p_{1}^{n} \\
\vdots & & \vdots \\
p_{1}^{1} & \cdots & p_{N}^{n}
\end{array}\right)=\left(\Delta p^{1}, \ldots, \Delta p^{n}\right)
$$

we have a linear multiple regression from $\Delta p$ to $\Delta q_{i}$, which can be completely summarized as:

$$
\Delta \mathbf{q}_{\mathbf{i}}=\boldsymbol{\epsilon}_{\mathbf{i}} \Delta \mathbf{p}+\mathbf{e r r}_{\mathbf{i}} \quad i=1, \ldots, N
$$

An unbiased estimator $\epsilon_{\mathbf{i}}$ would be the least square estimator $\hat{\epsilon}_{\mathrm{i}}$ :

$$
\hat{\epsilon}_{\mathbf{i}}=\left(\Delta \mathbf{p}^{\mathbf{T}} \Delta \mathbf{p}\right)^{-1} \Delta \mathbf{p}^{\mathbf{T}} \Delta \mathbf{q}_{\mathbf{i}}
$$

However, since the prices are defined relative to the mean price, the matrix $\Delta \mathbf{p}$ has only $N-1$ independent rows. The classical least squares estimation cannot be applied to matrices less than full row rank $N$ [30]. This problem can be overcome by using the Moore-Penrose pseudo inverse:

$$
\Delta \mathbf{p}^{+}=V S^{+} U^{T}
$$

with $\Delta \mathbf{p}=U S V^{T}$ the singular value decomposition of $\Delta \mathbf{p}$. The estimator $\hat{\boldsymbol{\epsilon}}_{\mathbf{i}}$ is then:

$$
\hat{\epsilon}_{\mathbf{i}}=\Delta \mathbf{p}^{+} \Delta \mathbf{q}_{\mathbf{i}}
$$

\section{Monte Carlo Simulation}

\section{A. Methodology}

The Monte Carlo technique is essentially a methodology that uses sample means to estimate population means [31]. Consider the function $z(x)$, which depends on a stochastic variable $x$ with a with a probability density function (PDF) $f(x)$. According to the Monte Carlo method, its population mean, or expected value can be approximated by the sample mean of $z$ :

$$
\bar{z}=\frac{1}{N_{m}} \sum_{i=1}^{N_{m}} z\left(x_{i}\right)
$$

where $x_{i}$ are $N_{m}$ randomly sampled values of the variable $x$ according to its PDF $f(x)$. It can be shown that $\lim _{N_{m} \rightarrow \infty} \bar{z}=$ $\langle z\rangle$. The standard error $\sigma(\bar{z})$ of the estimate of $\langle z\rangle$ can be approximated by [32]:

$$
\sigma(\bar{z}) \approx \frac{s(z)}{\sqrt{N_{m}}} \approx \sqrt{\frac{\overline{z^{2}}-\bar{z}^{2}}{N_{m}}}
$$

with $\bar{z}$ as in (13), $s(z)$ the sample standard deviation and $\overline{z^{2}}=$ $\left(1 / N_{m}\right) \sum_{i=1}^{N_{m}} z\left(x_{i}\right)^{2}$. This standard error converges to zero with a rate $\sim \sqrt{\left(1 / N_{m}\right)}$.

A Monte Carlo simulation can be seen as a stochastic simulation of a model with a source of randomness. The result of a simulation is then a value $z\left(x_{i}\right)$, which is used in (13) to estimate the population mean $\langle z\rangle$.

\section{B. Application}

The model for flexible residential demand (see Section II) contains numerous stochastic elements, listed in Table I. This means that each generated neighborhood is different, and thus also the corresponding elasticity matrix for a neighborhood. The whole process can be thought of as a simulation with stochastic variables $x$. To be able to make conclusions for a whole region consisting of a lot of different neighborhoods, the average behaviour of these neighborhoods has to be known. 
This can be found by applying a Monte Carlo simulation on the model. The results from the simulation - the reference electricity use $q_{r e f}$ and the elasticity matrix $\epsilon_{N \times N}-$ are then the dependent values $z(x)$.

By performing the same simulation for different neighbourhoods, one can obtain different samples $z\left(x_{i}\right)$ of these values. Applying equation (13) on the samples gives a Monte Carlo estimate of the real average value. In order to obtain a good estimate, a sufficient amount of neighborhoods has to be created. However, performing the regression on a neighborhood in itself is already very computationally expensive, so again a compromise has to be made. If around $N_{m}=100$ simulations are performed then the standard error of the mean $\sigma(\bar{z}) \approx s(z) / \sqrt{(100)}=0.10 \cdot s(z)$ is brought down to $10 \%$ of its standard deviation.

\section{RESULTS}

The Monte Carlo method is applied to 100 neighborhoods for an average day of each season, both for weekdays and weekend days.

\section{A. Reference Scenario}

As stated in Section III, the elasticities are defined w.r.t. a certain reference point $\left(p_{r e f}, q_{r e f}\right)$. The reference price profile $p_{r e f}$ is taken to be an average of all daily Belpex [29] prices of 2012 and 2013 (see Figure 1).

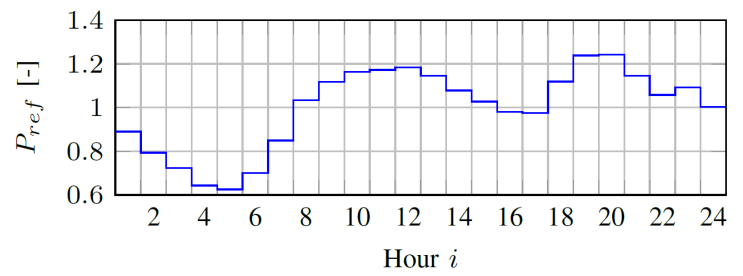

Fig. 1. Reference price profile

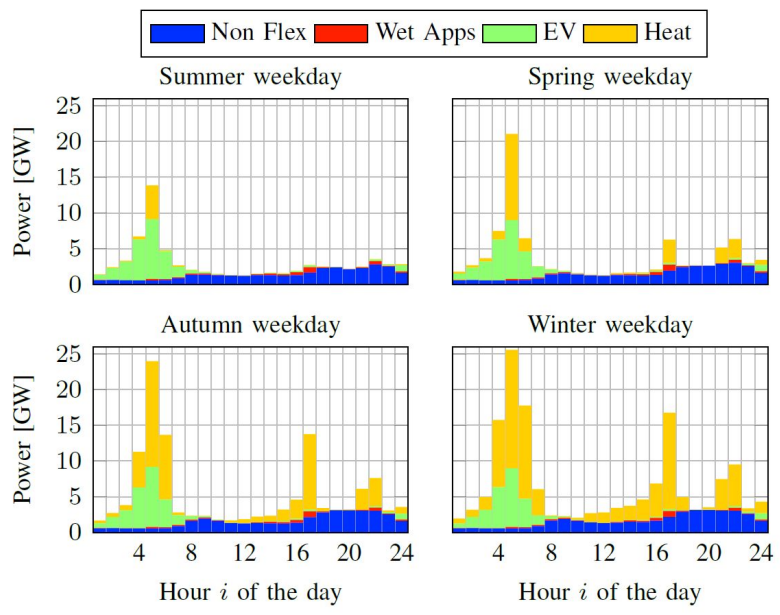

Fig. 2. Reference residential electricity consumption scaled up to the country level of Belgium for a weekday in summer, spring, autumn and winter
The corresponding average electricity consumption is found by applying the Monte Carlo method to the electricity consumption of 100 neighborhoods, when they receive the price signal $p_{\text {ref }}$. Since this is an estimate of the average over all possible neighborhoods, these results can be scaled up to the country level of Belgium.

1) Average Annual Electricity Consumption: The average annual electricity consumption of a household is more than twice that of a current average Belgian household (9 128 kWh/year vs. $4387 \mathrm{kWh} / \mathrm{year}$ ). This can be explained by the extensive electrification assumed in the model: all heating is electric and $71.4 \%$ of the households possess an EV (see Table II).

2) Reference Electricity Consumption Profile: Starting from the reference price profile, the model calculates a reference electricity consumption profile (see Figure 2). In this profile the peak power demand is found to increase by a factor 5 to 8 compared to today. The combination of automatic demand response and a RTP scheme leads to significant load syncing in the cheapest hours.

However, it must be noted here that it is not the goal of this model to predict the shape of the residential electricity consumption profile, but only to quantify the available flexibility. The reference electricity consumption profile will merely be the starting point for any changes in demand triggered by a difference between the actual price and the reference price profile. The magnitude of these changes will depend on the price differences and the relevant price elasticities. An example is given in Section V-B.

Thus, the reference electricity consumption profile shown here will not be the final electricity consumption profile. In practice, the reference price profile, the reference electricity consumption profile and the price elasticities that come out of this model will be used in e.g. an investment planning model or a unit commitment model. In such models a feedback on the price is included, which will limit the actual power peaks, as these would cause a significant upward price correction. These peaks, therefore, must not happen, but give an indication of the power that can be shifted if desired. Note that at present grid constraints are not considered. These would limit the maximum achievable power shift. Further research is needed to study the influence of these constraints.

TABLE II

ANNUAL AVERAGE RESIDENTIAL ELECTRICITY CONSUMPTION FOR A HOUSEHOLD SUBJECT TO THE REFERENCE PRICE PROFILE

\begin{tabular}{lr}
\hline Type of demand & Electricity consumption $(\mathrm{kWh} / \mathrm{yr})$ \\
\hline Non-flexible & 3091 \\
Electric heating & 3587 \\
Electric vehicle & 2146 \\
Wet appliances & 304 \\
Total & $\mathbf{9 1 2 8}$ \\
\hline
\end{tabular}




\section{B. Price Elasticities}

In order to get an estimate of the average elasticity matrix, the Monte Carlo method is applied to the elasticity matrices of the 100 neighborhoods. Figure 3 shows a heat map of the resulting average elasticity matrices for an average weekday in all four seasons.

The calculated price elasticities are quite large, meaning that a small change in price invokes a large change in demand. Automatic demand response combined with an RTP scheme appears to be very sensitive to price changes. This is something that should be taken into account when such a strategy would be adopted to control the flexibility of residential demand. Especially when used to e.g. perform a day-ahead planning of demand, power adjustments could be much larger than intended if insufficient real-time feedback is provided.

The largest elasticities, in absolute terms, are found in winter. This is the result of the increased level of operation of the heat pumps and electric heaters, which are therefore capable of providing more flexibility. This shows both the importance of thermal loads for the flexibility of residential demand and the strong influence of weather conditions on the calculated price elasticities. In warmer climates, where less heating is needed in winter, but air conditioning represents a significant load in summer, most flexbility might actually be available in summer.
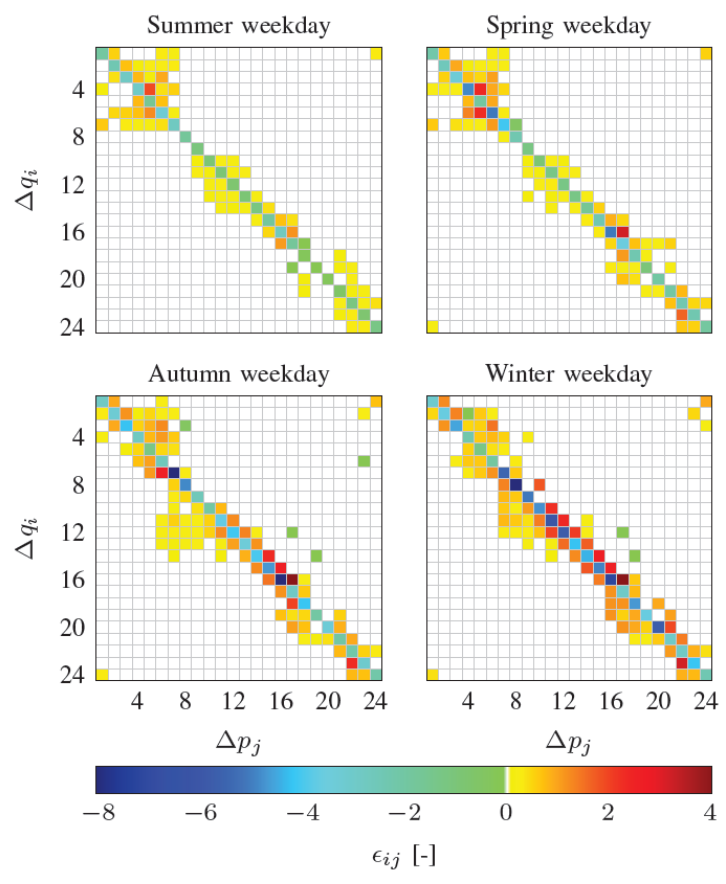

Fig. 3. Monte Carlo estimates of the mean price elasticity matrices for a weekday in summer, spring, autumn and winter

1) Own Elasticities: The diagonal values, or the own elasticities, are negative, meaning that a price increase in hour $i$ will result in a demand decrease in that same hour $i$. The largest values are found at hour 7, 8 and hour 16 . However, since these values are determined relative to the reference electricity consumption $q_{r e f, i}$, these hours do not necessarily coincide with the hours where most flexibility is available in absolute terms. Figure 4 shows the absolute shift in electricity demand $q_{i}-q_{r e f, i}$ for a relative decrease in the electricity price $\Delta p_{i}$ of $1 \%$ at every hour $i$ w.r.t. the reference price profile. The greatest shifts in demand occur in the early morning around hour 5 and in the afternoon around hour 17. The flexibility available in the early morning comes from the charging of the EVs and the heating of the households before breakfast. The flexibility in the afternoon comes from the possible anticipated heating of the households for when the residents return home.

Little difference is observed between weekdays and weekend days. Finally, when temperatures and solar irradiance decrease, flexibility increases, confirming the aforementioned influence of the weather conditions.

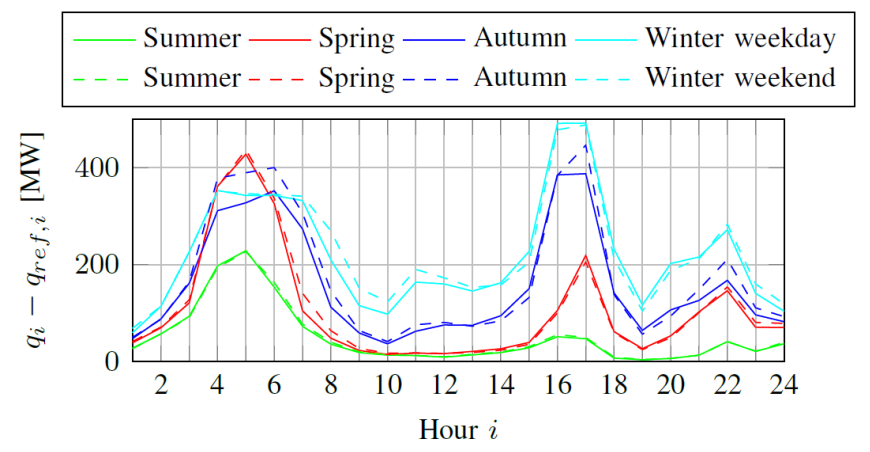

Fig. 4. Absolute shift in electricity consumption with a price decrease of $\Delta p_{i}=-1 \%$ at every hour $i$ for all seasons, on weekdays and weekend days, scaled up to the Belgian level

2) Cross Elasticities: The off-diagonal values, or cross elasticities, are almost all positive. They allow to determine how electricity consumption is shifted over time. An example is presented in Figure 5 for a price decrease of $30 \%$ in hours 7 and 15 . The electricity consumption in those hours is seen to increase, whereas it decreases in the adjacent hours. Looking at the elasticity matrices in Figure 3, the furthest shift in demand that is observed is 7 hours. In general, electricity consumption can be displaced between the early morning and the first hours of the night and between the evening and the end of the afternoon.

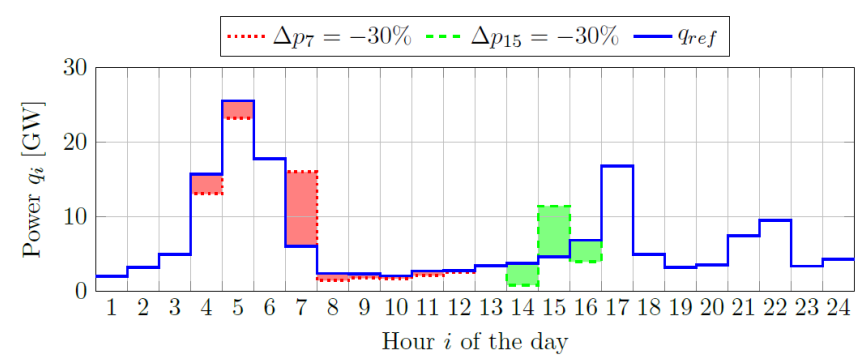

Fig. 5. New electricity consumption with a price change of $-30 \%$ at hour 7 , and at hour 15 , starting from the reference electricity consumption of an average winter weekday, scaled up to the Belgian level 


\section{CONCLUSION}

This paper presented a method to quantify the flexibility of automatic demand response combined with real time pricing applied to residential electricity demand using price elasticities. The reference price profile, the reference electricity consumption profile and the price elasticities that come out of the model can be used in e.g. an investment planning model or a unit commitment model to represent the flexibility of residential electricity demand.

This method is applied to an extensively electrified scenario in 2050 in Belgium. The average annual residential electricity consumption is found to double compared to current consumption levels. The results show that, when neglecting grid constraints, power peaks in demand can be incurred that are 5 to 8 times larger than the peaks observed today. The calculated price elasticities are quite large, indicating that automatic demand response combined with an RTP scheme is very sensitive to price changes. Furthermore, the resulting price elasticities are strongly influenced by weather conditions. For the Belgian case a higher amount of flexibility is available in winter than in summer due to increased operation of the heat pumps. In warmer climates, where e.g. the use of air conditioning in summer is significant and there is less need for heating in winter, this trend may be inversed.

Future work will include studying the influence of grid constraints on the available flexibility and the integration of the price elasticities in an investment model to evaluate the impact of the flexibility of residential demand on the optimal generation portfolio.

\section{REFERENCES}

[1] European Climate Foundation, "Roadmap 2050: A practical guide to a prosperous, low-carbon Europe: Policy Recommendations," European Climate Foundation, Tech. Rep., Apr. 2010. [Online]. Available: http://www.roadmap2050.eu/

[2] European Commission, "Communication from the Commission to the Council, the European Parliament, the European Economic and Social Committee and the Committee of the Regions - Energy Roadmap 2050," Dec. 2011.

[3] Eurelectric, "Power Choices - Pathways to Carbon-Neutral Electricity in Europe by 2050," Union of the Electricity Industry - EURELECTRIC, Brussels, Tech. Rep., Nov. 2009. [Online]. Available: www.eurelectric. org/PowerChoices2050/

[4] L. Söder, H. Abildgaard, A. Estanqueiro, C. Hamon, H. Holttinen, E. Lannoye, E. Gómez-Lázaro, M. O’Malley, and U. Zimmermann, "Experience and challenges with short-term balancing in European systems with large share of wind power," IEEE Transactions on Sustainable Energy, vol. 3, pp. 853-861, 2012.

[5] A. van Stiphout, K. Poncelet, K. De Vos, and G. Deconinck, "The impact of operating reserves in generation expansion planning with high shares of renewable energy sources," in 14th IAEE European Energy Conference, 2014, pp. 1-15.

[6] M. H. Albadi and E. F. El-Saadany, "Demand Response in Electricity Markets: An Overview," in 2007 IEEE Power Engineering Society General Meeting, pp. 1-5.

[7] E. Bloustein, Assessment of customer response to real time pricing, Rutgers - The State Universtity of New Jersey, Tech. Rep, Jun. 2005. [Online]. Available: http://ceeep.rutgers.edu/wp-content/uploads/2013/ 11/customerresponse.pdf

[8] C. Nabe, G. Papaefthymiou, and A. Mullane, "All island renewable grid study updated to include demand side management," Ecofys Germany GmbH, Tech. Rep., Mar. 2009. [Online]. Available: http://www.ecofys.com/en/publication/ all-island-renewable-grid-study-updated-to-include-demand-side-manag/
[9] D. Devogelaer, J. Duerinck, D. Gusbin, Y. Marenne, W. Nijs, M. Orsini, and M. Pairon, "Towards 100\% renewable energy in Belgium by 2050," VITO, ICEDD, Federal Planning Bureau, Brussels, Tech. Rep., Dec. 2012. [Online]. Available: http://www.plan.be/publications/Publication_ det.php?lang=n1\&TM=30\&IS=63\&KeyPub=1191

[10] F. A. Farret and M. G. Simões, Eds., Integration of Alternative Sources of Energy. Hoboken, NJ, USA: John Wiley \& Sons, Inc., Dec. 2005.

[11] S. Koch, J. L. Mathieu, and D. S. Callaway, "Modeling and Control of Aggregated Heterogeneous Thermostatically Controlled Loads for Ancillary Services," in Proc. PSCC, 2011, pp. 1-7.

[12] W. Zhang, J. Lian, C.-Y. Chang, K. Kalsi, and Y. Sun, "Reduced-order modeling of aggregated thermostatic loads with demand response," in 2012 IEEE 51st IEEE Conference on Decision and Control (CDC), pp. $5592-5597$.

[13] M. Alizadeh, A. Scaglione, and R. J. Thomas, "From Packet to Power Switching: Digital Direct Load Scheduling," IEEE Journal on Selected Areas in Communications, vol. 30, no. 6, pp. 1027-1036, Jul. 2012.

[14] N. Leemput, F. Geth, B. Claessens, J. Van Roy, R. Ponnette, and J. Driesen, "A case study of coordinated electric vehicle charging for peak shaving on a low voltage grid," in 2012 3rd IEEE PES Innovative Smart Grid Technologies Europe (ISGT Europe), pp. 1-7.

[15] C. De Jonghe, "Short-Term Demand Response in Electricity Generation Planning and Scheduling - Facilitating wind power integration," Ph.D. dissertation, KU Leuven, 2011.

[16] A. Faruqui and S. Sergici, "Household response to dynamic pricing of electricity: a survey of 15 experiments," Journal of Regulatory Economics, vol. 38, no. 2, pp. 193-225, Aug. 2010.

[17] I. Richardson, M. Thomson, D. Infield, and A. Delahunty, "A modelling framework for the study of highly distributed power systems and demand side management," in 2009 International Conference on Sustainable Power Generation and Supply, pp. 1-6.

[18] S. Faberi, R. Stamminger, W. Mebane, and R. Esposito, "Preparatory Studies for Eco-design Requirements of EuPs Part I PRESENT SITUATION Task 2 : Economic and Market Analysis," EuP-Netzwerk, Tech. Rep., Nov. 2007. [Online]. Available: http://www.landtechnik.uni-bonn.de/forschung/ haushaltstechnik/publikationen/report-to-eup-lot-13-task-3-ht-30

[19] K. Bruninx, D. Patteeuw, E. Delarue, L. Helsen, and W. D'Haeseleer, "Short-term demand response of flexible electric heating systems: The need for integrated simulations," in 2013 10th International Conference on the European Energy Market (EEM), 2013, pp. 1-10.

[20] T. Van Oevelen, "Control of heat pump systems in residential buildings, implementation of model-based predictive control," Ph.D. dissertation, KU Leuven, 2008.

[21] Meteotest, "Meteonorm." [Online]. Available: http://meteonorm.com/

[22] IEE Project EPISCOPE, "TABULA." [Online]. Available: http: //www.building-typology.eu/building-typology/country/be/

[23] F. Peuser, K.-H. Remmers, and M. Schnauss, Solar Thermal Systems Successful Planning and Construction. Beuth Verslag GmbH, 2002.

[24] International Energy Agency, "Technology Roadmap: Electric and Plugin Hybrid Vehicles (EV/PHEV)," Paris, France, Tech. Rep.

[25] "Mobility report of Flanders," Mobiliteitsraad van Vlaanderen, Tech. Rep., Sep. 2009. [Online]. Available: http://www.mobiliteitsraad.be/ sites/default/files/documenten/pdfpublicaties/1556.pdf

[26] J. Van Roy, N. Leemput, S. De Breucker, F. Geth, P. Tant, and J. Driesen, "An Availability Analysis and Energy Consumption Model for a Flemish Fleet of Electric Vehicles," in European Electric Vehicle Congress (EEVC), 2011.

[27] Belgian FPS Economy, "Population structure by households per year, region and size." [Online]. Available: http://statbel.fgov.be/nl/statistieken/ cijfers/bevolking/structuur/huishoudens/jaar_gewest_grootte/

[28] E. Veldman, "Power play: impacts of flexibility in future residential electricity demand on distribution network utilisation," Ph.D. dissertation, Technische Universiteit Eindhoven, 2013.

[29] Belpex SA, "Belgian Power Exchange." [Online]. Available: https: //www.belpex.be/

[30] L. Fahrmeir, T. Kneib, S. Lang, and D. Marx, Regression: Models, Methods and Applications. Springer, 2013.

[31] W. L. Dunn and J. K. Shultis, Exploring Monte Carlo Methods. Elsevier, 2011.

[32] C. Lemieux, Monte Carlo and Quasi-Monte Carlo Sampling. Springer, 2009. 